Full length article

\title{
Minimum toe clearance and tripping probability in people with unilateral transtibial amputation walking on ramps with different prosthetic designs
}

\author{
Mauricio Riveras $^{\mathrm{a}, \mathrm{b}}$, Emiliano Ravera ${ }^{\mathrm{a}, \mathrm{b}}$, David Ewins ${ }^{\mathrm{c}, \mathrm{d}}$, Aliah F. Shaheen ${ }^{\mathrm{d}, \mathrm{e}}$, \\ Paola Catalfamo-Formento ${ }^{\mathrm{a}, \mathrm{b}, \mathrm{d}, *}$ \\ ${ }^{a}$ Human Movement Research Laboratory, Facultad de Ingeniería, Universidad Nacional de Entre Ríos, Oro Verde, Entre Ríos, Argentina \\ ${ }^{\mathrm{b}}$ Instituto de Investigación en Bioingeniería y Bioinformática, CONICET-UNER, Oro Verde, Entre Ríos, Argentina

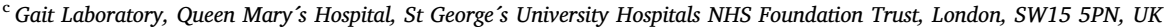 \\ ${ }^{\mathrm{d}}$ Centre for Biomedical Engineering, Department of Mechanical Engineering Sciences, University of Surrey, Guildford, GU2 7XH, UK \\ ${ }^{\mathrm{e}}$ Department of Life Sciences, Brunel University, London, Uxbridge UB8 3PH, UK
}

\section{A R T I C L E I N F O}

\section{Keywords:}

Gait analysis

Hydraulic prosthetic foot with microprocessor control

Energy storage and return prosthetic foot Prosthetic safety

Amputee gait.

\begin{abstract}
A B S T R A C T
Background: Minimum Toe Clearance (MTC) is defined as the minimum vertical distance between the lowest point under the front part of the foot and the ground, during mid-swing. Low values of MTC and walking on inclines are both related to higher probability of tripping and falling in lower limb amputees. New prosthetic designs aim at improving MTC, especially on ramps, however the real effect on MTC still needs investigation. The objective of this study was then to evaluate the effect of different prosthetic designs on MTC in inclined walking.

Methods: Thirteen transtibial amputees walked on a ramp using three different prostheses: non articulating ankle (NAA), articulating hydraulic ankle (AHA), and articulating hydraulic ankle with microprocessor (AHA-MP). Median MTC, coefficient of variation (CV), and tripping probability (TP) for obstacles of 10 and $15 \mathrm{~mm}$ were compared across ankle type in ascent and descent.

Findings: When using AHA-MP, larger MTC median values for ascending $(\mathrm{P} \leq 0.001, \mathrm{~W}=0.58)$ and descending the $\operatorname{ramp}(\mathrm{P}=0.003, \mathrm{~W}=0.47)$ were found in the prosthetic limb. Also significantly lower $\mathrm{CV}$ was found on the prosthetic limb for both types of AHA feet when compared to NAA for descending the ramp $(\mathrm{P}=0.014, \mathrm{~W}=$ 0.45). AHA-MP showed the lowest TP for the prosthetic leg in three conditions evaluated. On the sound limb results showed the median $\mathrm{MTC}$ was significantly larger $(\mathrm{P}=0.009, \mathrm{~W}=0.43)$ and $\mathrm{CV}$ significantly lower $(\mathrm{P}=$ $0.005, \mathrm{~W}=0.41$ ) when using an AHA in ascent.

Interpretation: Both AHA prosthetic designs help reduce the risk of tripping of the prosthetic limb by increasing the median MTC, lowering its variability and reducing TP for both legs when ascending and descending the ramp. For most of the conditions, AHA-MP showed the lowest TP values. Findings suggest that AHA prostheses, especially AHA-MP could reduce the risk of tripping on ramps in amputees.
\end{abstract}

\section{Introduction}

There is no a unique definition of Minimum Toe Clearance (MTC). One definition commonly used describes it as the minimum vertical distance between the lowest point under the front part of the shoe or foot and the ground and occurs during the mid-swing phase of the gait cycle [1-5]. The risk of falling after tripping at the time of MTC increases because of the close distance between the foot and the floor at the time, the high velocity of the foot during swing and the limited compensatory mechanisms available (when compared to tripping with the heel or midsole) [6]. What is more, research has found that elderly subjects with a fall history demonstrated lower MTC than elderly people without history of falling [7] and also participants with lower limb amputation who reported one or more trip-related stumbles showed a lower MTC compared to participants who reported zero triprelated stumbles on the prosthetic side [8]. Hence, poor control of this parameter could increase the risk of tripping and the associated likelihoods of falling [6,8-10].

Research on MTC in unimpaired subjects suggests that it is affected by several factors such as age, cognitive and sensory conditions [9], [11-13], type of terrain [14,15] and type of shoes [15]. The changes are reflected on MTC distribution, including median, interquartile

\footnotetext{
* Corresponding author at: Human Movement Research Laboratory, Facultad de Ingeniería, Universidad Nacional de Entre Ríos, Oro Verde, Entre Ríos, Argentina.

E-mail address: pcatalfamo@ingenieria.uner.edu.ar (P. Catalfamo-Formento).
} 
Table 1

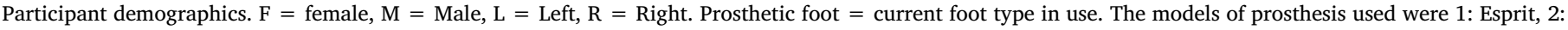
Oseoreflex, 3: Flex foot-Elation, 4: Echelon, 5: Elan.

\begin{tabular}{|c|c|c|c|c|c|c|c|}
\hline Subject & Height (m) & Mass (kg) & Age (years) & Time using prosthesis (years) & Prosthetic foot & Gender & Amputated side \\
\hline 1 & 1.76 & 71 & 51 & 22 & $\mathrm{AHA}^{4}$ & M & $\mathrm{L}$ \\
\hline 2 & 1.64 & 65 & 23 & 0.4 & $\mathrm{NAA}^{1}$ & $\mathrm{~F}$ & $\mathrm{R}$ \\
\hline 3 & 1.67 & 51 & 41 & 3 & $\mathrm{AHA}^{4}$ & M & $\mathrm{R}$ \\
\hline 4 & 1.79 & 73 & 22 & 2,7 & $\mathrm{AHA}^{4}$ & $\mathrm{~F}$ & $\mathrm{~L}$ \\
\hline 5 & 1.85 & 90 & 30 & 1.3 & $\mathrm{AHA}^{4}$ & M & $\mathrm{R}$ \\
\hline 6 & 1.73 & 68 & 43 & 9 & $\mathrm{NAA}^{1}$ & M & $\mathrm{R}$ \\
\hline 7 & 1.75 & 86.5 & 41 & 5 & AHA-MP ${ }^{5}$ & M & $\mathrm{L}$ \\
\hline 8 & 1.69 & 60 & 23 & 4 & AHA-MP ${ }^{5} / \mathrm{AHA}^{4}$ & M & $\mathrm{R}$ \\
\hline 9 & 1.88 & 82 & 34 & 18 & $\mathrm{NAA}^{1}$ & M & $\mathrm{R}$ \\
\hline 10 & 1.74 & 80 & 66 & 11 & $\mathrm{NAA}^{1}$ & M & $\mathrm{R}$ \\
\hline 11 & 1.85 & 87 & 28 & 17 & $\mathrm{NAA}^{2}$ & M & $\mathrm{R}$ \\
\hline 12 & 1.73 & 57 & 51 & 47 & $\mathrm{NAA}^{3}$ & $\mathrm{~F}$ & $\mathrm{~L}$ \\
\hline 13 & 1.80 & 106 & 45 & 0.3 & $\mathrm{AHA}^{4}$ & M & $\mathrm{R}$ \\
\hline
\end{tabular}

range, skewness and kurtosis [11-13]. Furthermore, it has been suggested that increasing median MTC, reducing MTC variability and increasing kurtosis and skewness are control strategies to avoid tripping [11].

In terms of pathological gait, unilateral transtibial amputees must make important adjustments to motor control on both the prosthetic and intact side to compensate for the absence of the limb and walk safely $[16,17]$. In particular, compensatory mechanisms such as hiphiking, vaulting, circumduction and increased knee flexion are used by amputees in order to increase foot clearance during prosthetic swing phase [18-21]. Despite this available mechanisms, people with amputation present a reduced MTC on the prosthetic limb compared with the intact limb when walking over level ground, with interlimb differences increasing when walking on an uneven surface [22]. The reduced MTC seen on the prosthetic side may be explained in part by the inability to actively dorsiflex the foot [23] and also by the increased energy required to use the compensatory mechanisms [21].

Prosthetic feet called energy storage and return feet typically incorporate flexible heel and forefoot keels. Their constructive characteristics make them capable of storing energy during loading response and mid-stance and returning a proportion of the stored energy at terminal stance and preswing to help with forward progression and push-off [23]. However, these prosthetic feet do not have an articulating ankle (from now on called non articulating ankle, NAA, prosthesis) and do not allow dorsiflexion of the foot during swing. Articulating hydraulic ankle (AHA) devices, instead, provide dampened stance-phase passive articulation and would allow the foot to passively dorsiflex during stance. This enables the foot to leave the ground in a relatively dorsiflexed position and remain so throughout the swing phase [23]. This would "raise the toes" during swing and thus increase MTC, which, as long as variability in MTC did not increase, would reduce the likelihood of tripping.

Rosenblatt et al. [24] compared MTC values for an AHA and an NAA foot on treadmill walking at 0 and $5 \%$ upwards inclination on eight transtibial amputees and found larger values of MTC for the AHA prosthesis for both inclinations. Also, Johnson et al. [23] found a significant increase in the mean MTC for both the prosthetic and intact limbs when using AHA compared to NAA, when walking on level ground. These results suggest that people with amputation might have a reduced risk of tripping when walking with AHA. However, withinparticipant variability in MTC, which has been reported to be a risk factor for falling, also increased on the prosthetic side when using AHA compared to NAA.

More recently, an articulating hydraulic ankle-foot device incorporating a microprocessor control (AHA-MP) has been introduced (Elan; Chas. A Blatchford and Sons, Ltd., Basingstoke, UK). This device has the functionality of the AHA but also incorporates sensors that determine the angle of the terrain being walked on. This information is used to alter the hydraulic damping to predefined settings so as to improve the foot-ground interaction for the current terrain [25]. A complete evaluation of the effect of this prosthesis on MTC is still pending.

Walking on ramps may present the greatest problem in falling risks compared to level ground and stairs [26]. What is more, falling and the fear of falling are pervasive among amputees [27]; more than $50 \%$ of subjects with unilateral amputation reported falling in the previous year, whereas $49.2 \%$ reported a fear of falling. The adaptations performed by amputees to diminish the risk of falling due to a low MTC have been shown to increase metabolic cost $[24,28]$. For these reasons new prosthetic designs should focus on increasing MTC and consequently diminishing the risk of falling. Hence the effect of different prosthetic design on MTC when walking on ramps is an area of interest.

The effect of using NAA, AHA and AHA-MP prostheses on the MTC of prosthetic and sound limb of transtibial amputees when ascending and descending a ramp has not been studied. Then, the aim of this study was to evaluate the effect of three different prosthetic designs (NAA, AHA, AHA-MP) on MTC and tripping probability (TP) when walking up and down a ramp.

\section{Methods}

\subsection{Participants}

Fourteen participants were involved in this study. Data from one of them had to be discarded due to loss of kinematic markers during the trials. The data obtained from the remaining thirteen physically active, unilateral transtibial amputees (mean (SD) age 38.23 (13.2) years, mass 75.1 (15.4) $\mathrm{kg}$, height $1.76(0.07) \mathrm{m}$ ) was analysed (Table 1). The causes of amputation in the study population were: traumatic (10 participants), collateral to diabetes disease (2 participants) and secondary to osteomyelitis (one participant). All of the participants had been using the prosthesis for at least four months prior to data capture (mean 10.8 (13.05) years, range 0.3--47 years). Each participant gave written informed consent prior to their involvement. The local ethics committee approval was obtained for the protocol.

\subsection{Prosthetic intervention and protocol}

\subsubsection{Prosthetic conditions}

Subjects walked up and down a ramp using three different prostheses: NAA, AHA and AHA-MP. The three devices were chosen from the Endolite family (Endolite, Chas. A. Blatchford and Sons Ltd., Basingstoke, UK). The NAA device used was an Esprit, the AHA an Echelon and the AHA-MP an Elan. The participants used first the prosthesis they were more unfamiliar with (either AHA or NAA). For example, if they habitually used a non articulating foot, then an AHA 
foot was fitted first. In this way, a minimum familiarization time of one hour with the non-habitual prosthesis, was ensured (while the researchers were performing other activities in the laboratory, such as setting up the terrain). It was considered that a longer period of adaptation time was preferred for the non-habitual prosthesis, rather than randomizing the use of the prosthesis, to ensure a minimum familiarization time as proposed in the literature [23,29-31].

The fitting of the prosthesis was performed by an experienced prosthetist, who ensured the best possible alignment for each foot. The same prosthetist was in charge of the set-up and alignment for all participants and he based his selections on his professional judgment and on his probed experience on the topic. The socket, suspension and alignment of the shank pylon were unchanged across foot types and each type of foot was attached to the distal end of the shank pylon with as close to the same alignment, total leg length and set-up as possible.

The settings that control the rates of articulation within the hydraulic foot (damping) for level ground (both for Echelon and Elan) and for ramps (Elan) were adjusted independently by the prosthetist until deemed to provide optimal function at self-selected, comfortable walking speed. In order to ensure that the working mode of Elan was adjusted as ramp up or down, it was manually set at the beginning of each trial using a Bluetooth connection with the foot's microprocessor [25].

\subsubsection{Data acquisition and processing}

Participants walked in a straight line along a $6 \mathrm{~m}, 5^{\circ}$ inclination ramp (Fig. 1a) at their freely-selected comfortable walking speed [32]. A minimum of 6 trials in ascent and 6 in descent were performed by each patient with each prosthesis. Kinematic data was recorded at 200 $\mathrm{Hz}$ using an eleven-camera motion capture system (ProReflex, Qualisys, Göteborg, Sweden). During data collection, participants wore their own flat-soled shoes.

Data from two retro-reflective markers of $9.5 \mathrm{~mm}$ diameter placed on the toe and equivalent location on the prosthetic side were used for this study. Additional markers were also placed on lower limbs (Fig. 1b). Raw kinematic data from the markers on the toe was processed using MatLab (R2016a, MathWorks Inc., Natick, Massachusetts, United States.). Each participant walked the ramp performing an average of 12 steps to walk up and another 12 to walk it down. Of those, six steps performed in the middle of the ramp, and included in the acquisition volume of the cameras, were analysed.

The estimation of the surface of the ramp and the MTC was performed following strategies proposed in the literature [5,8,18,33,34]. The minimum position of the toe marker during each cycle time was detected. This position occurs just before toe off and it represents the closest position of the toe marker to the ground. The line connecting these positions during the trial was used to estimate the surface of the ramp (see dash line in Fig. 1c). For each gait cycle, a local minimum of the vertical position of the trajectory of the marker placed on the toe (minl, in Fig.1c) occurring between two local maximum values (MAXl, in Fig. 1c) was detected. Minimum toe clearance was then calculated as the vertical distance between the trajectory of the marker placed on the toe and the ramp surface at the time of this local minimum (MTC in Fig. 1c).

Tripping probability (TP) is the parameter used to quantify the risk of tripping or falling [24]. In order to improve the precision of the TP estimation, a function that best fitted the MTC experimental data obtained for each participant in each experimental condition was used. For this research, the Empirical Cumulative Distribution Function (ECDF) was used to represent the MTC distribution by randomly generated 10,000 data points that follow a Pearson distribution. The Pearson distribution was used due to its flexibility since it comprises a wide range of distributions, and it adjusts appropriately to unknown ones [35]. In order to estimate the best fit, four descriptive parameters (or moments) of the measured MTC were used: mean, standard deviation, skewness and kurtosis (Fig. 3a). The ECDF was generated then for each trial, each participant and each condition and was used to calculate the probability of tripping. Following a similar approach to other studies $[24,36]$, the tripping probability was calculated for the case in which objects not seen by the person, appeared in the walking pathway at the time of the MTC. Then two hypothetical height of objects (or thresholds) of 10 and $15 \mathrm{~mm}$ were considered, as a compromise between the probability of not seeing the object and the risk of tripping with it $[37,38]$. Finally, the tripping probability was calculated from the ECDF, using these thresholds.

\subsection{Statistical analyses}

Descriptive statistical analysis was performed for the MTC data from individual participant. In line with the results of other studies in the literature, [11,12,36,39,40] a non-normal MTC distribution was assumed. For this reason, the total median, first quartile (q1), third quartile (q3), coefficient of variation (CV), skewness (s) and kurtosis (k) were calculated and used to compare the MTC for the three prosthesis, in each condition (walking up and down the ramp, for the prosthetic and sound limb). In order to statistically compare MTC and TP data between the prosthesis the non-parametric Friedman test and the Dunn-Bonferroni post hoc adjustment for multiple comparisons [41] were applied using SPSS (23.0.0.0, IBM, Armonk New York U.S.A.). The alpha level was set at 0.05 . The effect size was calculated using the Kendall's W coefficient [42]. Values of W was interpreted as follows: $<0.11$, very weak; $0.11-0.30$, weak; $0.31-0.50$, moderate; $0.51-0.70$, strong; and $>0.71$, very strong effect $[43,44]$.

\section{Results}

Fig. 2 shows MTC histograms for all participants with a fixed number of data sample, the histograms share some common characteristics with participants' individual histograms: they deviate from a normal distribution to a greater or lesser extent and most of them show a right skew $(s>0)$.

Table 2 shows the median, coefficient of variation (CV) for MTC, Skewness, Kurtosis and per stride tripping probability (TP) of striking a hypothetical, unseen obstacle of a given height (10 or $15 \mathrm{~mm}$ ), for the prosthetic and sound limb, when using NAA, AHA and AHA-MP prosthetic foot and for ascending and descending the ramp.

The results of MTC median, CV, skewness and kurtosis, that reached statistical significant levels between the prosthetic feet (Table 2) are shown in Fig. 3. In particular, Fig. 3a shows the boxplot of the MTC median for those conditions that showed a statistical significant difference and Fig. 3b shows the boxplot of the MTC coefficient of variation for those walking conditions that showed statistically significant differences.

The results show that the median values of MTC when ascending the ramp are smaller, and hence less safer, than the ones for descending it (except on the sound limb when using AHA). This could be expected since the upward inclination of the ground diminishes the separation between the ground and the foot during the swing phase. Also the CV was larger for ascending the ramp. And, in terms of the probability of tripping, as it was expected, the probability of striking an object while walking up the ramp is larger than while walking down the ramp, and this occurs for both the amputee and sound side.

Fig. 4 shows the results obtained for Tripping Probability. As an example, Fig. 4a shows the ECDF for the prosthetic side of one participant while walking up the ramp. It is possible to see how the probability is calculated for each prostheses and each obstacle. For example, for the $10 \mathrm{~mm}$ threshold, only NAA and AHA prosthesis showed a TP different from zero. Fig. $4 \mathrm{~b}$ shows the boxplots of the tripping probability for those walking conditions that showed statistically significant differences between prosthesis. 

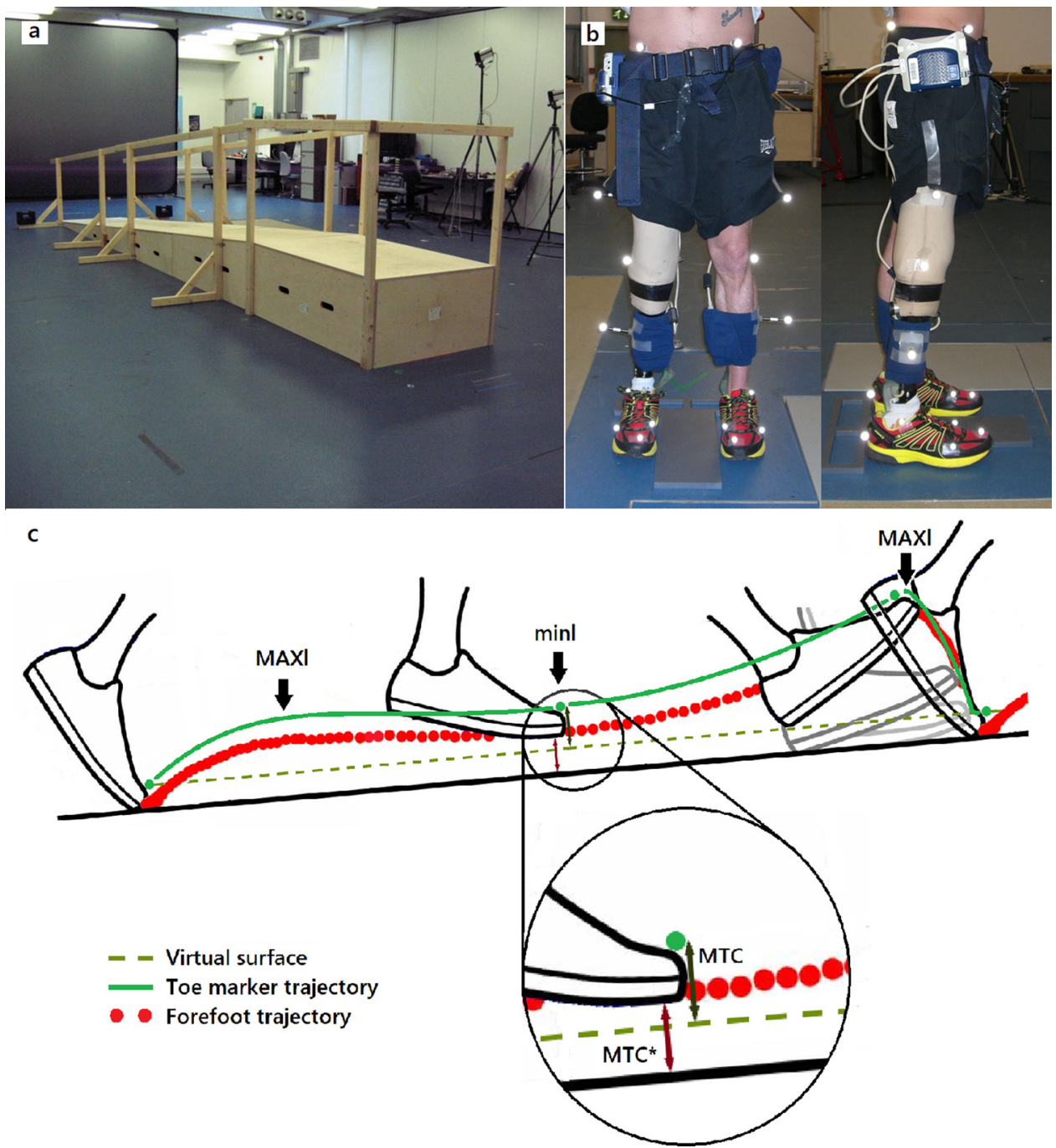

Fig. 1. Data collection protocol. a) Set up of the ramp (modular design). The inclined walk way was $6 \mathrm{~m}$ long, $1 \mathrm{~m}$ wide and $5^{\circ}$ inclination. It was custom made and incorporated a raised surface at its upper end to provide a stable area for resting and turning. b) Marker set used in the study. c) Estimation of the walking surface and MTC. MTC*: MTC according to definition, MTC: the value of MTC calculated in this study, MAXl: local maximum and minl: local minimum.

\section{Discussion}

Based on MTC data alone, it has been suggested that there are three possible strategies to avoid tripping: (a) to increase median MTC; (b) to reduce MTC variability; and (c) to increase kurtosis and skewness [11].

In terms of the median MTC, the results of the present study showed that for the prosthetic side, the median values of MTC were significantly larger when using both types of AHA feet than the NAA foot for ascending and descending the $\operatorname{ramp}(\mathrm{P}=0.03$ with moderate size effect and $\mathrm{P}=0.01$ and strong size effect, respectively) (Fig. 2.a). These results are in agreement with previous research comparing an AHA (without microprocessor control) with an NAA [23,24]. The present results provide the additional information that AHA-MP showed the largest group median for both conditions.

Regarding the coefficient of variation, a measure of the variability of the MTC values, it was significantly lower for both types of AHA feet than NAA for descending the ramp $(P=0.014$, with moderate size effect) for the prosthetic side (Fig. 2.b). And AHA-MP presented the lowest CV for both conditions (descending and ascending the ramp).

Finally, in relation to skewness and kurtosis, a positive skewness would suggest that more steps include higher, and hence safer, values of MTC while higher levels of kurtosis would imply greater number of steps for which the MTC values are concentrated on the median and therefore lower risk of stumbling. The results of this study showed that the MTC distribution when participants used an AHA-MP prosthesis presented significantly larger values of skewness when compared to AHA prosthesis (Fig. 2c) and significantly larger values of kurtosis when compared to the NAA prosthesis for the prosthetic limb when descending the ramp (Fig. 2d). Also, in general, the MTC distribution when participants used an AHA-MP prosthesis presented larger skewness and kurtosis, for both ascending and descending the ramp.

Then, the results of this study showed that both AHA prostheses and AHA-MP in particular, aid on the three strategies for avoiding tripping on the prosthetic side. What is more, AHA-MP showed significantly lower median per stride tripping probability than NAA for ascending and descending the ramp for the prosthetic limb (Fig. 3b). As mentioned before, the reduced MTC seen on the prosthetic side is probably due, at least in part, to the inability to actively dorsiflex the foot during the swing phase [23]. AHA devices present their maximum dorsiflexion at the time of toe off, which reduces the angle of plantarflexion throughout the swing phase $[23,31]$. This hydraulic mechanism may compensate in part for the lack of active dorsiflexion during swing and explain the larger values of MTC found for both AHA prostheses. What is more, the AHA-MP prosthesis allows for a set-up of the plantarflexion and dorsiflexion resistance which can be different for ascending and descending the ramp. This could influence the degree of dorsiflexion 
A

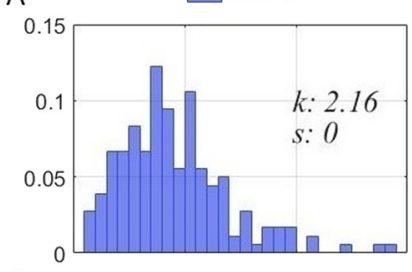

$\mathrm{B}$
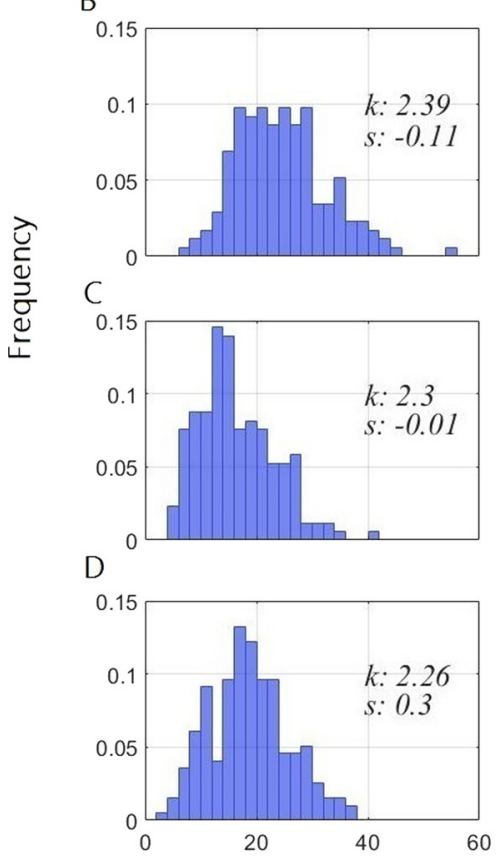

AHA
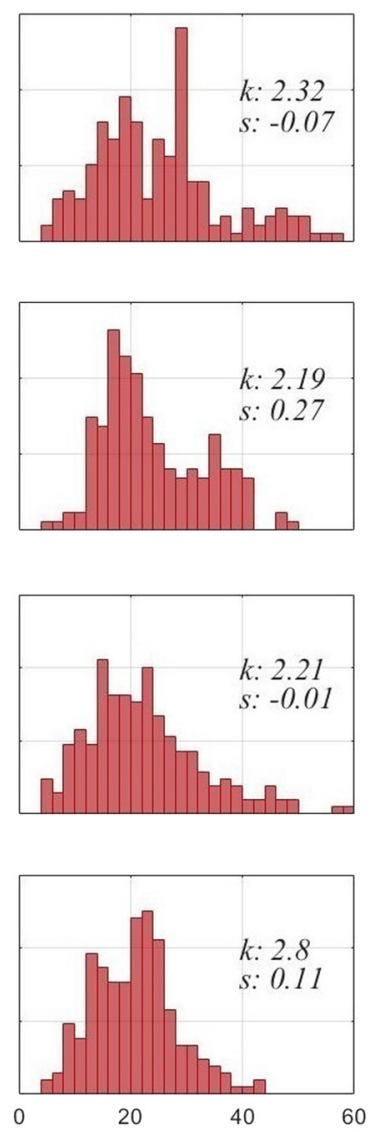

AHA-MP
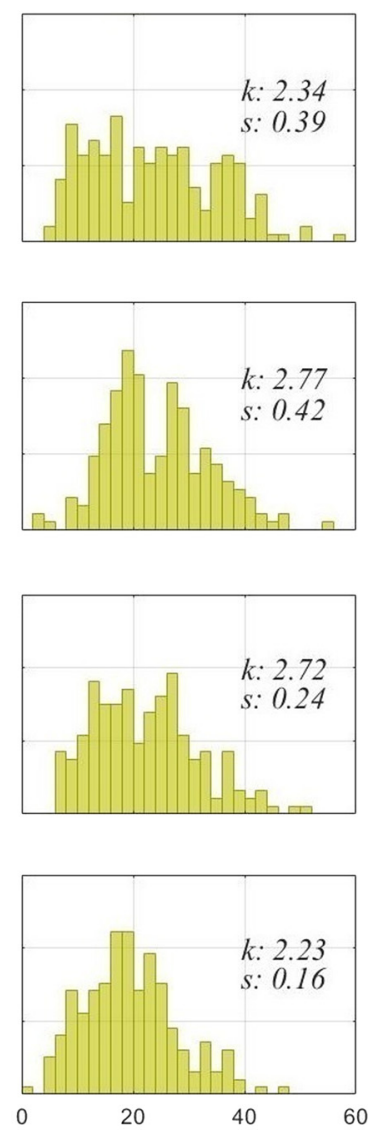

Minimum Toe Clearance ( $\mathrm{mm})$

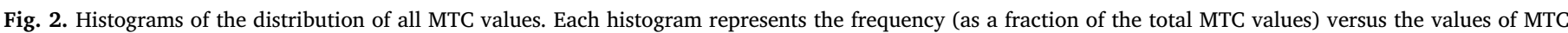

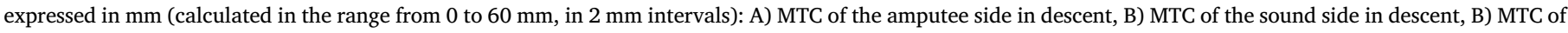
the amputee side in ascent and D) MTC of thesound side in ascent. $\mathrm{k}=$ kurtosis, $\mathrm{s}=$ skewness.

reached at the point of toe off and hence moderate the degree of dorsiflexion during swing phase. And this could have improved the values of skewness for the AHA-MP prosthesis.

On the sound side, the results using different prosthesis did not show a clear trend for all conditions. AHA-MP showed median MTC values in between those found for AHA and NAA (both AHA-MP and NAA showed significantly lower values when compared to AHA, Fig. 2a) and CV which were in the middle of the three for descending the ramp and the largest for ascending it (Fig. 2b). Contrary to results presented from subjects walking on level ground, in general, median values of MTC were smaller for the sound limb than for the prosthetic limb and the variability was higher for both AHA prostheses. These results could suggest that there is a level of instability of the prosthetic limb during single support that could affect the swing phase of the sound limb $[9,45,46]$. Future research should analyse the effect of stability on the values of MTC of the sound side.

Some limitations of this work should be considered. Firstly, the order of presentation of the prosthetic feet was not randomized. It was considered that a longer period of adaptation time was preferred for the non-habitual prosthesis, rather than randomizing the use of the prosthesis. However, this could imply that differences in MTC may reflect an ordering effect.

Secondly, the method used here to estimate the MTC considers the trajectory of a marker placed on the toe rather than the actual difference between the sole of the shoe and the floor (see Fig. 1). The surface of the ramp is estimated by a line connecting the final contact points of the toe (and hence the absolute minimum position of the toe during each gait cycle), as proposed by Rosenblatt et al. [24]. The MTC is then calculated as the distance between a local minimum of the marker during mid-swing and this estimated surface. This is done under the assumption that this distance is equal to the one between the lowest point under the front part of the shoe and the ground. However, changes in the distance between the toe marker and the front of the shoe and the deformation of the shoe during the support phase of foot may challenge the assumption. More studies are necessary to estimate the induced error [47]. Nevertheless, if shoe deformation and relative distance of the toe marking with respect to the sole are considered constant for the same patient independently of the prosthesis used, then this error is minimized. It could then be considered as an appropriate method for experiments investigating changes in MTC [48].

Finally, the tripping probability here was calculated for the hypothetical case of the presence of an unseen object that hits the foot at the exact time of the occurrence of the MTC. This is clearly an unluckily scenario. The probability of tripping with an object of the same height as the ones considered here would be lower if the person can see it and activate the compensatory mechanisms and it would also be lower if it does not appear at the exact time of MTC. However, it is used here as in other studies [24,36], as a resource to study the behavior of different prosthesis. Even when the scenario presented here may not be frequently present in real life, the tendency in the behavior of the prosthesis can be studied by using it. 


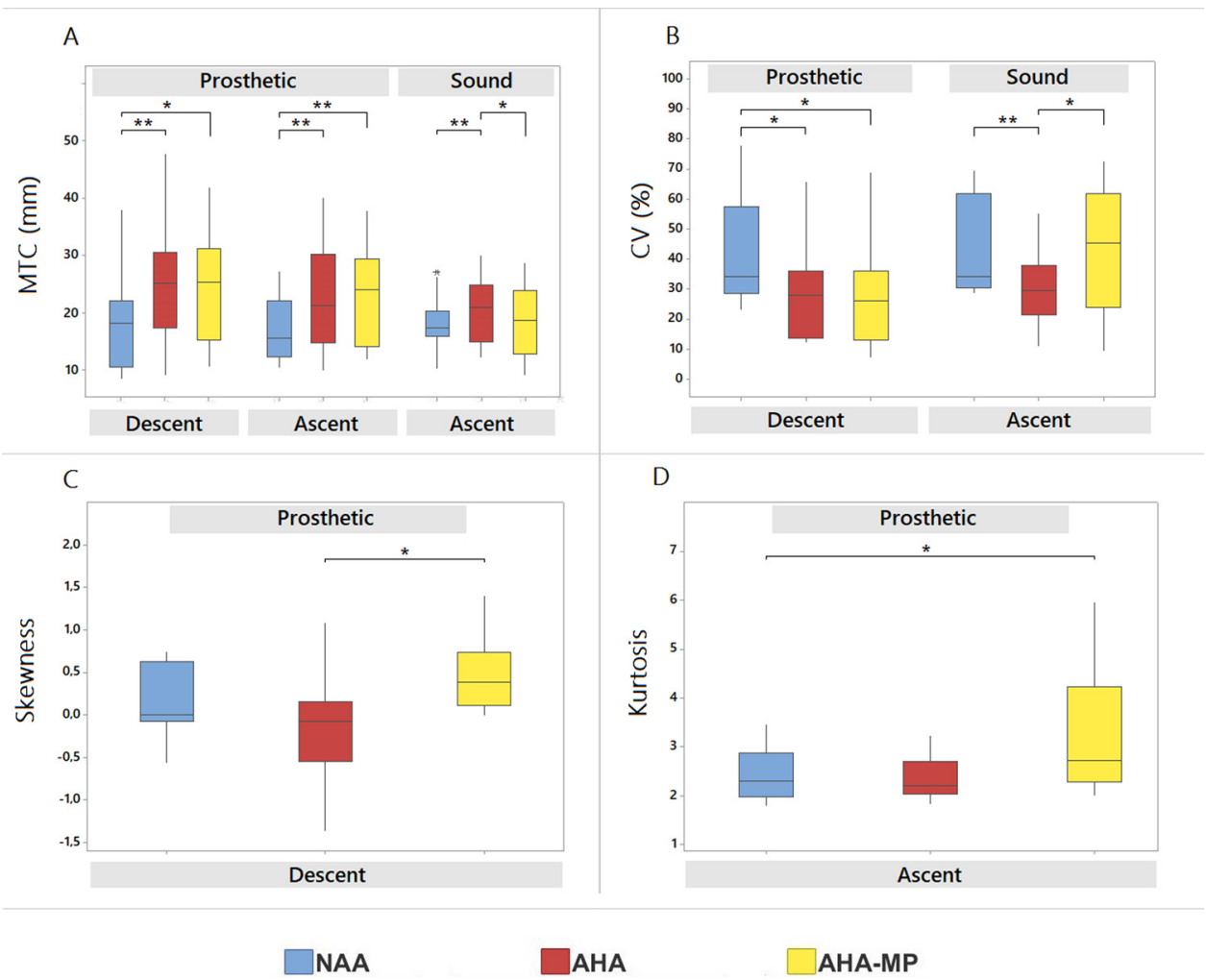

Fig. 3. Conditions that showed statistical significant differences between the prosthetic feet in Table 2gr3

a) MTC median. b) Coefficient of variation (CV) of MTC c) Skewness d) Kurtosis * $(P<0.05)$, ** $(P<0.01)$.

Table 2

Group median (q1 q3), Coefficient of variation (CV) (q1 q3), Skewness (q1 q3) and Kurtosis (q1 q3) in minimum toe clearance (MTC) for the prosthetic and sound limb, when using NAA, AHA and AHA-MP prosthetic foot. Median (q1 q3) per-stride probability of striking a hypothetical, unseen obstacle of a given height (10 and $15 \mathrm{~mm})$. W: Kendall's W coefficient.

\begin{tabular}{|c|c|c|c|c|c|c|c|c|c|c|c|}
\hline & & \multicolumn{5}{|c|}{ Prosthetic limb } & \multicolumn{5}{|l|}{ Sound limb } \\
\hline & & \multicolumn{3}{|l|}{ Prosthesis } & \multirow[t]{2}{*}{$\mathrm{P}$ value } & \multirow[t]{2}{*}{ W } & \multirow{2}{*}{$\begin{array}{l}\text { Prosthesis } \\
\text { NAA }\end{array}$} & \multirow[b]{2}{*}{ AHA } & \multirow[b]{2}{*}{ AHA-MP } & \multirow[t]{2}{*}{$P$ value } & \multirow[t]{2}{*}{ W } \\
\hline & & NAA & AHA & AHA-MP & & & & & & & \\
\hline \multirow[t]{6}{*}{ Descent } & $\begin{array}{l}\text { Median } \\
(\mathrm{mm})\end{array}$ & $\begin{array}{l}18 \\
(1122)\end{array}$ & $\begin{array}{l}25 \\
(18 \text { 31) }\end{array}$ & $\begin{array}{l}25 \\
(1531)\end{array}$ & 0.003 & 0.47 & $\begin{array}{l}23 \\
(1930)\end{array}$ & $\begin{array}{l}20 \\
(1931)\end{array}$ & $\begin{array}{l}20 \\
(1929)\end{array}$ & 0.305 & 0.09 \\
\hline & CV (\%) & $\begin{array}{l}34 \\
(2958)\end{array}$ & $\begin{array}{l}28 \\
(1436)\end{array}$ & $\begin{array}{l}26 \\
(1336)\end{array}$ & 0.014 & 0.33 & $\begin{array}{l}26 \\
(2131)\end{array}$ & $\begin{array}{l}31 \\
(1743)\end{array}$ & $\begin{array}{l}29 \\
(16 \quad 39)\end{array}$ & 0.146 & 0.16 \\
\hline & Skewness & $\begin{array}{l}0.0 \\
(-0.10 .6)\end{array}$ & $\begin{array}{l}-0.1 \\
(-0.50 .2)\end{array}$ & $\begin{array}{l}0.4 \\
\left(\begin{array}{ll}0.1 & 0.7\end{array}\right)\end{array}$ & 0.037 & 0.25 & $\begin{array}{l}-0.1 \\
(-0.30 .5)\end{array}$ & $\begin{array}{l}0.3 \\
(-0.20 .5)\end{array}$ & $\begin{array}{l}0.4 \\
(-0.11 .2)\end{array}$ & 0.500 & 0.05 \\
\hline & Kurtosis & $\begin{array}{l}2.2 \\
(1.72 .7)\end{array}$ & $\begin{array}{l}2.3 \\
(1.83 .1)\end{array}$ & $\begin{array}{l}2.3 \\
(2.23 .3)\end{array}$ & 0.368 & 0.08 & $\begin{array}{l}2.4 \\
(2.23 .0)\end{array}$ & $\begin{array}{l}2.2 \\
(1.82 .7)\end{array}$ & $\begin{array}{l}2.8 \\
(2.24 .1)\end{array}$ & 0.092 & 0.18 \\
\hline & TP 10 (\%) & $\begin{array}{l}2 \\
(046)\end{array}$ & $\begin{array}{l}- \\
(06)\end{array}$ & $\begin{array}{l}- \\
(012)\end{array}$ & 0.261 & 0.10 & $\begin{array}{l}- \\
(0\end{array}$ & $\begin{array}{l}- \\
\left(\begin{array}{ll}0 & 0\end{array}\right)\end{array}$ & $\begin{array}{l}- \\
(0\end{array}$ & 0.204 & 0.08 \\
\hline & TP 15 (\%) & $\begin{array}{l}29 \\
(381)\end{array}$ & $\begin{array}{l}8 \\
(0 \quad 32)\end{array}$ & $\begin{array}{l}- \\
(048)\end{array}$ & 0.006 & 0.40 & $\begin{array}{l}2 \\
(018)\end{array}$ & $\begin{array}{l}7 \\
(024)\end{array}$ & $\begin{array}{l}4 \\
(024)\end{array}$ & 0.975 & 0.00 \\
\hline \multirow[t]{6}{*}{ Ascent } & $\operatorname{Median}(\mathrm{mm})$ & $\begin{array}{l}16 \\
(1222)\end{array}$ & $\begin{array}{l}21 \\
(1530)\end{array}$ & $\begin{array}{l}24 \\
(1529)\end{array}$ & 0.001 & 0.58 & $\begin{array}{l}17 \\
(1621)\end{array}$ & $\begin{array}{l}21 \\
(1525)\end{array}$ & $\begin{array}{l}19 \\
(1324)\end{array}$ & 0.009 & 0.43 \\
\hline & CV (\%) & $\begin{array}{l}42 \\
(2651)\end{array}$ & $\begin{array}{l}33 \\
(2945)\end{array}$ & $\begin{array}{l}29 \\
(1439)\end{array}$ & 0.070 & 0.21 & $\begin{array}{l}34 \\
(3162)\end{array}$ & $\begin{array}{l}29 \\
(2238)\end{array}$ & $\begin{array}{l}45 \\
(2462)\end{array}$ & 0.009 & 0.41 \\
\hline & Skewness & $\begin{array}{l}0 \\
(-0.2 \quad 0.3)\end{array}$ & $\begin{array}{l}0 \\
(-0.5 \\
(0.2)\end{array}$ & $\begin{array}{l}0.2 \\
(-0.40 .6)\end{array}$ & 0.584 & 0.04 & $\begin{array}{l}0.3 \\
(0.2 \quad 0.6)\end{array}$ & $\begin{array}{l}0.1 \\
(-0.70 .9)\end{array}$ & $\begin{array}{l}0.2 \\
(-0.30 .4)\end{array}$ & 0.292 & 0.10 \\
\hline & Kurtosis & $\begin{array}{l}2.3 \\
\left(\begin{array}{ll}2.0 & 2.9\end{array}\right)\end{array}$ & $\begin{array}{l}2.2 \\
(2.02 .7)\end{array}$ & $\begin{array}{l}2.7 \\
(2.34 .2)\end{array}$ & 0.023 & 0.29 & $\begin{array}{l}2.3 \\
(2.02 .8)\end{array}$ & $\begin{array}{l}2.8 \\
(2.63 .5)\end{array}$ & $\begin{array}{l}2.2 \\
(2.03 .2)\end{array}$ & 0.050 & 0.23 \\
\hline & ТP 10 (\%) & $\begin{array}{l}15 \\
(033)\end{array}$ & $\begin{array}{l}1 \\
(019)\end{array}$ & $\begin{array}{l}- \\
(09)\end{array}$ & 0.014 & 0.45 & $\begin{array}{l}10 \\
\left(\begin{array}{ll}0 & 19\end{array}\right)\end{array}$ & $\begin{array}{l}- \\
(017)\end{array}$ & $\begin{array}{l}2 \\
(0 \quad 30)\end{array}$ & 0.629 & 0.19 \\
\hline & TP 15 (\%) & $\begin{array}{l}45 \\
(1675)\end{array}$ & $\begin{array}{l}9 \\
(050)\end{array}$ & $\begin{array}{l}2 \\
(062)\end{array}$ & 0.000 & 0.61 & $\begin{array}{l}36 \\
(047)\end{array}$ & $\begin{array}{l}12 \\
(152)\end{array}$ & $\begin{array}{l}19 \\
(161)\end{array}$ & 0.570 & 0.04 \\
\hline
\end{tabular}




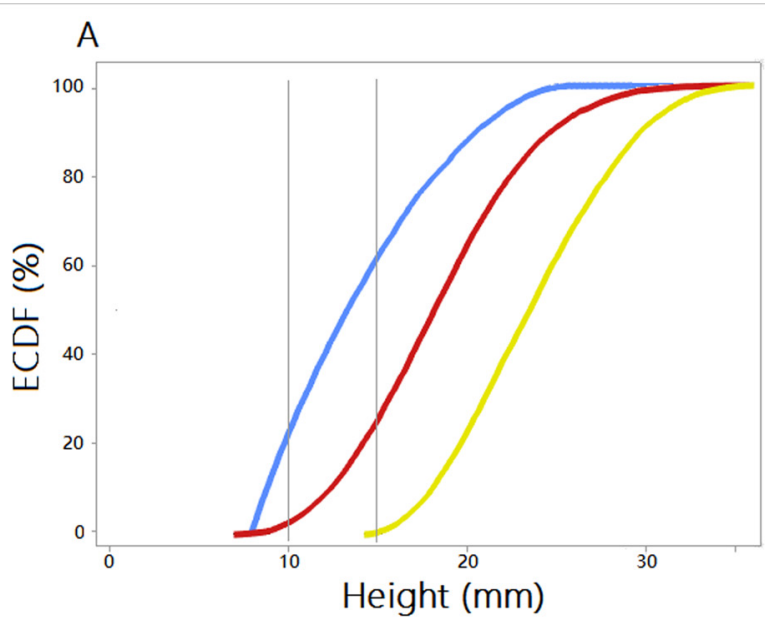

B

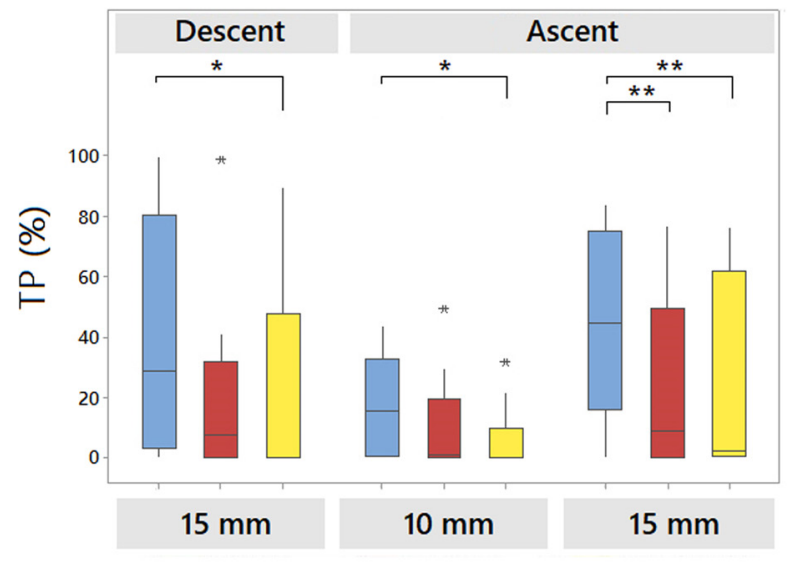

NAA $\square$ AHA $\square$ AHA-MP

Fig. 4. a) Tripping probability modelling for one amputee using NAA, AHA and AHA-MP prosthesis, on prosthetic side during ramp ascent. Briefly, per-stride probabilities of striking a hypothetical, unseen obstacle of a given height (10 and $15 \mathrm{~mm}$ in vertical gray lines), are obtained from the intersection of these lines with the curves obtained from the estimated cumulative distribution. b) Tripping probability for those walking conditions that showed statistically significant differences. * $(\mathrm{P}<0.05), * *(\mathrm{P}<0.01)$.

\section{Conclusion}

This is the first study that evaluates the effect of using NAA, AHA and AHA-MP prostheses on the MTC of prosthetic and sound limb of transtibial amputees when ascending and descending a ramp. Given the risks of falling associated with incline walking, and the energetic cost of diminishing these risks, prosthetic feet that could lower the probability of tripping are desirable. The results of this study showed that AHA-MP prosthetic feet showed larger median MTC, lower CV, increased positive skewness and increased kurtosis on the prosthetic side when compared to NAA. Also, the probability of striking the obstacle when using an AHA-MP foot was null for both obstacles in descending the ramp and for the $10 \mathrm{~mm}$ obstacle in ascending the ramp, for prosthetic limb. For the sound side, the results using different prosthesis were variable. Results suggest that AHA-MP aids on strategies for avoiding tripping.

\section{Source of funding}

This study was partly supported by the Universidad Nacional de Entre Ríos (National University of Entre Ríos, Argentina) and CONICET
Argentina (National Scientific and Technical Research Council).

\section{Declaration of Competing Interest}

The authors declare not having any financial and personal relationships with other people or organisations that could inappropriately influence (bias) their work.

\section{Acknowledgements}

This study was made possible by the support of the University of Entre Ríos (UNER) and CONICET, Argentina.

\section{References}

[1] A.R. de Asha, J.G. Buckley, The effects of walking speed on minimum toe clearance and on the temporal relationship between minimum clearance and peak swing-foot velocity in unilateral trans-tibial amputees, Prosthet. Orthot. Int. 39 (2) (2015) $120-125$.

[2] B.W. Schulz, Minimum toe clearance adaptations to floor surface irregularity and gait speed, J. Biomech. 44 (7) (2011) 1277-1284.

[3] T. Maruyama, S. Kanai, H. Date, Tripping risk evaluation system based on human behavior simulation in laser-scanned 3D as-is environments, Autom. Constr. 85 (January) (2018) 193-208.

[4] D.T.H. Lai, R.K. Begg, S. Taylor, M. Palaniswami, Detection of tripping gait patterns in the elderly using autoregressive features and support vector machines, J. Biomech. 41 (January 8) (2008) 1762-1772.

[5] J.C. Menant, J.R. Steele, H.B. Menz, B.J. Munro, S.R. Lord, Effects of walking surfaces and footwear on temporo-spatial gait parameters in young and older people, Gait Posture 29 (April 3) (2009) 392-397.

[6] H. Chen, J. Ashton-Miller, N. Alexander, A. Schultz, Stepping over obstacles: gait parameters of young and old adults, J. Gerontol. 46 (6) (1991) M196-203.

[7] E.C. Cebolla, A.L.F. Rodacki, P.C.B. Bento, Balance, gait, functionality and strength: comparison between elderly fallers and non-fallers, Brazilian J. Phys. Ther. 19 (2) (2015) 146-151.

[8] N.J. Rosenblatt, A. Bauer, M.D. Grabiner, Relating minimum toe clearance to prospective, self-reported, trip-related stumbles in the community, Prosthet. Orthot. Int. 41 (4) (2017) 387-392.

[9] P.M. Mills, R.S. Barrett, S. Morrison, Toe clearance variability during walking in young and elderly men, Gait Posture 28 (July 1) (2008) 101-107.

[10] A.H. Khandoker, S.B. Taylor, C.K. Karmakar, R.K. Begg, M. Palaniswami, Investigating scale invariant dynamics in minimum toe clearance variability of the young and elderly during treadmill walking, IEEE Trans. Neural Syst. Rehabil. Eng. 16 (4) (2008) 380-389.

[11] R. Begg, R. Best, L. Dell'Oro, S. Taylor, Minimum foot clearance during walking: strategies for the minimisation of trip-related falls, Gait Posture 25 (February 2) (2007) 191-198.

[12] W.A. Sparrow, R.K. Begg, S. Parker, Variability in the foot-ground clearance and step timing of young and older men during single-task and dual-task treadmill walking, Gait Posture 28 (November 4) (2008) 563-567.

[13] V. Graci, D.B. Elliott, J.G. Buckley, Peripheral visual cues affect minimum-footclearance during overground locomotion, Gait Posture 30 (October 3) (2009) 370-374.

[14] A.H. Khandoker, C.K. Lynch K Fau-Karmakar, R.K. Karmakar Ck Fau - Begg, M. Begg Rk Fau - Palaniswami, M. Palaniswami, Toe clearance and velocity profiles of young and elderly during walking on sloped, J. Neuroeng. Rehabil. 7 (2010) 18.

[15] S.B. Thies, R.K. Jones, L.P.J. Kenney, D. Howard, R. Baker, Effects of ramp negotiation, paving type and shoe sole geometry on toe clearance in young adults, J. Biomech. 44 (October 15) (2011) 2679-2684.

[16] E.C. Prinsen, M.J. Nederhand, J.S. Rietman, Adaptation strategies of the lower extremities of patients with a transtibial or transfemoral amputation during level walking: a systematic review, Arch. Phys. Med. Rehabil. 92 (8) (2011) 1311-1325.

[17] D.J. Sanderson, P.E. Martin, Lower extremity kinematic and kinetic adaptations in unilateral below-knee amputees during walking, Gait Posture 6 (2) (1997) $126-136$.

[18] D.A. Winter, Foot trajectory in human gait: a precise and multifactorial motor control task, Phys. Ther. 72 (1) (1992) 45-53.

[19] R. Baker, A. Esquenazi, M.G. Benedetti, K. Desloovere, Gait Analysis : Clinical Facts, August (2016), pp. 560-574.

[20] L. Fradet, M. Alimusaj, F. Braatz, S.I. Wolf, Biomechanical analysis of ramp ambulation of transtibial amputees with an adaptive ankle foot system, Gait Posture 32 (June 2) (2010) 191-198.

[21] Sa. Gard, Use of quantitative gait analysis for the evaluation of prosthetic walking performance, JPO J. Prosthetics Orthot. 18 (2006) P93-P104.

[22] D.H. Gates, J.B. Dingwell, S.J. Scott, E.H. Sinitski, J.M. Wilken, Gait characteristics of individuals with transtibial amputations walking on a destabilizing rock surface, Gait Posture 36 (1) (2012) 33-39.

[23] L. Johnson, A.R. De Asha, R. Munjal, J. Kulkarni, J.G. Buckley, Toe clearance when walking in people with unilateral transtibial amputation: Effects of passive hydraulic ankle, Journal of Rehabilitation Research and Development 51 (3) (2014) $429-438$. 
[24] N.J. Rosenblatt, A. Bauer, D. Rotter, M.D. Grabiner, Active dorsiflexing prostheses may reduce trip-related fall risk in people with transtibial amputation, J. Rehabil. Res. Dev. 51 (8) (2014) 1229-1242.

[25] V. Struchkov, J.G. Buckley, Biomechanics of ramp descent in unilateral trans-tibial amputees: comparison of a microprocessor controlled foot with conventional anklefoot mechanisms, Clin. Biomech. Bristol Avon (Bristol, Avon) 32 (December) (2015) 164-170.

[26] R.C. Sheehan, J.S. Gottschall, At similar angles, slope walking has a greater fall risk than stair walking, Appl. Ergon. 43 (3) (2012) 473-478.

[27] W.C. Miller, M. Speechley, B. Deathe, The prevalence and risk factors of falling and fear of falling among lower extremity amputees, Arch. Phys. Med. Rehabil. 82 (8) (2001) 1031-1037.

[28] A.R. Wu, A.D. Kuo, Determinants of preferred ground clearance during swing phase of human walking, J. Exp. Biol. 219 (19) (2016) 3106-3113.

[29] X. Zhang, G. Fiedler, Z. Liu, Evaluation of gait variable change over time as transtibial amputees adapt to a new prosthesis foot, Biomed Res. Int. 2019 (i) (2019).

[30] A.B. Wanamaker, R.R. Andridge, A.M.W. Chaudhari, When to biomechanically examine a lower-limb amputee: a systematic review of accommodation times, Prosthet. Orthot. Int. 41 (5) (2017) 431-445.

[31] C.Y. Ko, et al., Comparison of ankle angle adaptations of prosthetic feet with and without adaptive ankle angle during level ground, ramp, and stair ambulations of a transtibial amputee: a pilot study, Int. J. Precis. Eng. Manuf. Technol. 15 (12) (2014) 2689-2693.

[32] A.R. De Asha, R. Munjal, J. Kulkarni, J.G. Buckley, Walking speed related joint kinetic alterations in trans-tibial amputees: impact of hydraulic 'ankle' damping, J. Neuroeng. Rehabil. 10 (1) (2013) 107.

[33] A. Merryweather, B. Yoo, D. Bloswick, Gait characteristics associated with trip-induced falls on level and sloped irregular surfaces, Minerals 1 (1) (2011) 109-121.

[34] D.H. Gates, J.M. Aldridge, J.M. Wilken, Kinematic comparison of walking on uneven ground using powered and unpowered prostheses, Clin. Biomech. Bristol Avon (Bristol, Avon) 28 (April 4) (2013) 467-472.

[35] H. Shore, Response modeling methodology: empirical modeling for engineering and science, Wiley Interdiscip. Rev. Comput. Stat. 3 (January 2005) (2005) 435.

[36] T. Killeen et al., Minimum toe clearance: Probing the neural control of locomotion
Sci. Rep.7, 1, 1-10, 2017.

[37] R. Best, R. Begg, A method for calculating the probability of tripping while walking, J. Biomech. 41 (January 5) (2008) 1147-1151.

[38] A.G. Byju, M.A. Nussbaum, M.L. Madigan, Alternative measures of toe trajectory more accurately predict the probability of tripping than minimum toe clearance, $\mathrm{J}$. Biomech. 49 (December 16) (2016) 4016-4021.

[39] A.R. De Asha, Variability and distribution of minimum toe clearance in individuals with unilateral transtibial amputation: the effects of walking speed, J. Prosthet. Orthot. 27 (3) (2015) 78-83.

[40] R.J. Best, R.K. Begg, L.D. Oro, Minimum foot clearance during walking: mean, sd, skewness and kurtosis. in: Gerber Hans, Roland Müller (Eds.), Congress of the International Society of Biomechanics, Book of Abstracts. International Society of Biomechanics, 2001, p. 88.

[41] O. J. Dunn, "Multiple comparisons using rank sums," Technometrics, vol 6 no. April 2013, pp. 37-41, 1964.

[42] M. Tomczak, E. Tomczak, The need to report effect size estimates revisited. An overview of some recommended measures of effect size, Trends Sport Sci. 1 (21) (2014) 19-25.

[43] C. Roy, Managing Delphi surveys using nonparametric statistical techniques, Decis Sci. 28 (3) (1997) 763-774.

[44] C.P. Orsbon, R.S. Kaiser, C.F. Ross, Physician opinions about an anatomy core curriculum: a case for medical imaging and vertical integration, Anat. Sci. Educ. 7 (4) (2014) 251-261.

[45] A.L. Hof, R.M. van Bockel, T. Schoppen, K. Postema, Control of lateral balance in walking. Experimental findings in normal subjects and above-knee amputees, Gait Posture 25 (2) (2007) 250-258.

[46] S. Wurdeman, J. Yentes, S. Myers, A. Jacobson, N. Stergiou, Both limbs in unilateral transtibial amputees display increased risk for tripping, Proc. 36th Annu. Meet. Am. Soc. Biomech. (2012) 15-18.

[47] J.K. Startzell, P.R. Cavanagh, A three-dimensional approach to the calculation of foot clearance during locomotion, Human Movement Science 18 (October 5) (1999) 603-611.

[48] K.L. Loverro, N.M. Mueske, K.A. Hamel, Location of minimum foot clearance on the shoe and with respect to the obstacle changes with locomotor task, J. Biomech. 46 (July 11) (2013) 1842-1850. 